\title{
Association of the negative anomalies of the quasistatic electric field in atmosphere with Kamchatka seismicity
}

\author{
S. Smirnov \\ Institute of Cosmophysical Research and Radio Wave Propagation of the Far-Eastern Branch of Russian Academy of Science, \\ Kamchatka region, Paratunka, Russia
}

Received: 29 May 2008 - Accepted: 2 July 2008 - Published: 25 July 2008

\begin{abstract}
One hundred three cases of a bay-like depression in the strength of the $E z$ component of the quasistatic electric field in the near-Earth atmosphere, observed from 1997 to 2002 on Kamchatka, have been analyzed statistically. It has been shown that the most probable length of a bay is 40-60 min. The most probable drops in $E z$ are minus 106$300 \mathrm{~V} / \mathrm{m}$. The dependence of these values on an earthquake class and a distance to the epicenter was not found. The probability of earthquake prediction over $24 \mathrm{~h}$ before an earthquake based on the $E z$ anomaly is $36 \%$.
\end{abstract}

\section{Introduction}

In different seismic regions of the world, such as Central Asia, Northern Caucasia and Transcaucasia, Kamchatka, Japan, China, etc., the anomalies in the daily variations in the quasistatic electric field in the near-Earth atmosphere were observed with different delay times the day before an earthquake (e.g. see Rulenko, 2000). This made it possible to use these anomalies to predict earthquakes and initiated intense studying these anomalies. However, these observations were either episodic or short-term. As a result, it was impossible to sort out characteristics of anomalous variations in $E z$ (such as their time profile and duration), the magnitude of deviation from the background level, and the dependence of these parameters on an earthquake class (magnitude, $\mathrm{M}$ ) and distance to the epicenter.

Institute of Cosmophysical Research and Radiowave Propagation (IKIR), Far East Division, Russian Academy of Sciences began in 1991 regular purposeful ground-based observations of the quasistatic electric field in the region of the Paratunka hydrothermal system on Kamchatka (Rulenko,

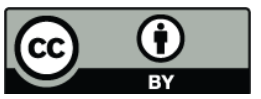

Correspondence to: S. Smirnov (sergey@ikir.ru)
1992). These studies are still continued at Paratunka geophysical observatory $\left(\phi=52^{\circ} 58.3^{\prime} \mathrm{N}, \lambda=158^{\circ} 14.9^{\prime} \mathrm{E}\right)$ (Buzevich et al., 1998a; Smirnov, 2002a). The first observations of the quasistatic electric field anomaly before the strong ( $M=6.1)$ earthquake are given in Rulenko (1992). It turned out that a negative $E z$ bay-like anomaly with a sharp leading front and subsequent smooth recovery to the background level over an hour and a half was observed a few hours before the earthquake on Kamchatka, as well as in Japan and China (Hao, 1998; Kondo, 1968). A similar character of the anomalous variation in $E z$ from several hours to a day before the earthquake was reported in the subsequent papers of the IKIR research group (Buzevich et al., 1998b, 2003; Mikhailov et al., 2002; Smirnov, 2001; Vershinin et al., 1999). At the same time, an anomalous positive burst of $E z$ before the quake with $\mathrm{M}=7.0$ was also detected (Rulenko et al., 1996).

The creation of the specialized soft-hardware complex (Buzevich et al., 1998a; Smirnov, 2002) made it possible to continuously and simultaneously register not only the quasistatic electric field but also geomagnetic, electromagnetic, and meteorological parameters necessary for detecting short-term and on-line earthquake precursors (Buzevich and Smirnov, 1998b). The first results of a ststistical analysis of the charactristics of anomalous $E z$ variations from 1997 to 2000 were published in the form of a brief communication (Smirnov, 2001). The results of similar studies performed from 1 January 1997, to 31 December 2002, are presented below. Preliminary results of these studies were published in Smirnov (2003).

The purpose of this paper is to perform a more complete statistical analysis of such $E z$ parameters as the magnitude of a negative anomaly, the anomaly duration, and the dependence of these parameters on an quake class (magnitude) and on a distance between an observation point and an earthquake epicenter. Only negative anomalies were considered, i.e. negative deviations of $E z$ from the positive diurnal behavior over $24 \mathrm{~h}$ before the quake.

Published by Copernicus Publications on behalf of the European Geosciences Union. 


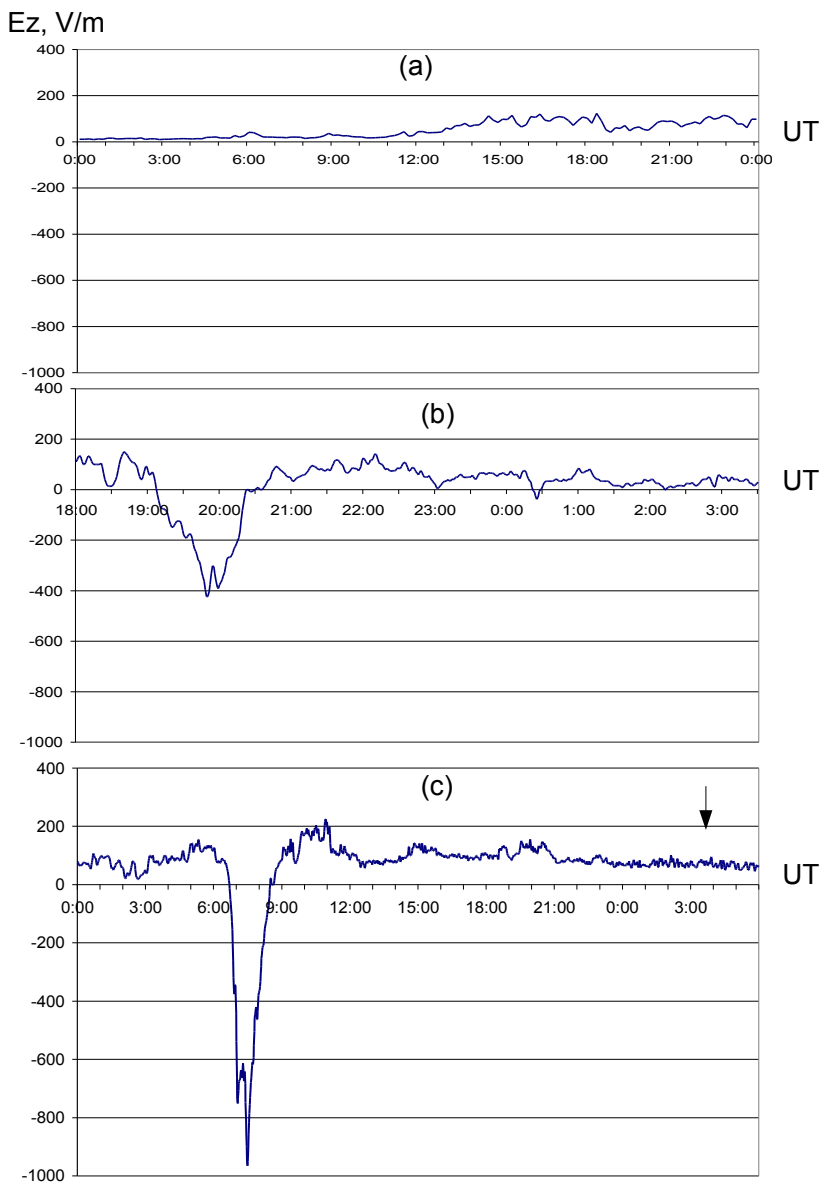

Fig. 1. Typical diurnal variations in the $E z$ field component during (a) days with fine weather and without anomalies, (b) days with precipitation, and (c) during days with fine weather but with an anomalous $E z$ behavior. The arrow indicates the earthquake instant.

\section{Method of registration}

The ground-based measurements of the quasistatic electric field $E z$ in the atmosphere are considered among very fine experiments. A diurnal behavior, except a UT-variation, is affected by different local sources including thunderstorms, low nimbostratus, precipitation in the form of rain and snow, hoarfrost, rime, and strong winds (Chernyavskii, 1937). Therefore, to distinguish seismic effects, it is necessary to measure $E z$ under the so-called normal conditions (moderate meteorological conditions) or under the conditions of fine weather in the absence of meteorological factors. Unfortunately, on Kamchatka even normal conditions are realized rarely due to its geographic location and closeness to the Pacific. The Gradient-M2 and Pole-2M flux meters of the soft-hardware complex were used at Paratunka observatory as sensors of the vertical component of the atmospheric quasistatic electric field. Ten-minute averaging was used in data sampling. The meteorological parameters (pressure, tem- perature, strength and direction of wind, precipitation, and cloudiness) were controlled simultaneously. Examples of diurnal $E z$ variations under the conditions of fine weather, as well as during precipitation, are presented in Buzevich et al. (1998b, 2003); Mikhailov et al. (2002, 2003). It follows from these papers that, under the conditions of fine weather, the diurnal $E z$ variations show a quiet background level of about $+(100-150) \mathrm{V} / \mathrm{m}$ at local night with an insignificant positive increase of $\Delta E \sim 100 \mathrm{~V} / \mathrm{m}$ in the evening and of up to (400-600) V/m in the morning. Rather chaotic positive and negative deviations of $E z$ (about 100-500) V/m from the mean diurnal behavior are possible during precipitation. More regular forms, when $E z$ crosses zero and reaches minus $(1000-1500) \mathrm{V} / \mathrm{m}$, are also possible. As was shown in the previous publications of measurements performed on Kamchatka, the anomalous variations in $E z$ before an earthquake most often display bay-like depressions in the field magnitude with zero crossing. Figure 1 shows typical diurnal variations in the $E z$ field component for (a) days with fine weather but without anomalies, (b) the days with precipitation, and (c) days with fine weather but with an anomalous $E z$ behavior frequently accompanied by earthquakes. The anomalies in the form shown in Fig. 1 appeared during the whole period of observations under consideration. Only the values of a decrease in the $E z$ field and the duration of a bay were different. For a further statistical analysis, we selected only data for days with fine weather. The anomalies with increased levels of $E z$ before and after the bay were excluded from an analysis. A situation was considered as an event when one or several earthquakes of classes $(\mathrm{K})$ from 11 to $15(\mathrm{M} \cong 4.7-6.7)$ occurred during $24 \mathrm{~h}$ after the anomaly, and the epicenters of these earthquakes were in the region with coordinates (44$55)^{\circ} \mathrm{N},(155-165)^{\circ} \mathrm{E}$, i.e. when the point of $E z$ observation was within the earthquake preparation zone.

\section{Main results}

From 1 January 1997, to 31 December 2002 (i.e. during 2189 days), we found out 103 cases of an anomalous behavior of the $E z$ component, and 409 earthquakes occurred in the above region. In 37 cases (36\%) the earthquakes really occurred within the next 1-24h. If we take the number 103 as a random sample, than the expected number of these events is $406 / 2189 \times 103=19$. Thus, the probability of earthquake occurrence $(36 \%)$ exceeds the random value by a factor of 1.9. Now, it is impossible to estimate the probability of event omission because the weather before many earthquakes was not fine. We constructed the distribution of the field strength anomalies based on a bay duration (Fig. 2a) and on a decrease in the field strength in the bay (Fig. 2b). The minimal magnitude of $E z$ in the sample with 10 min averaging was taken as a decrease in the field strength in the bay. The anomaly duration was determined by level equal to 0.9 of the bay minimum. Figure 2 indicates that the most probable 

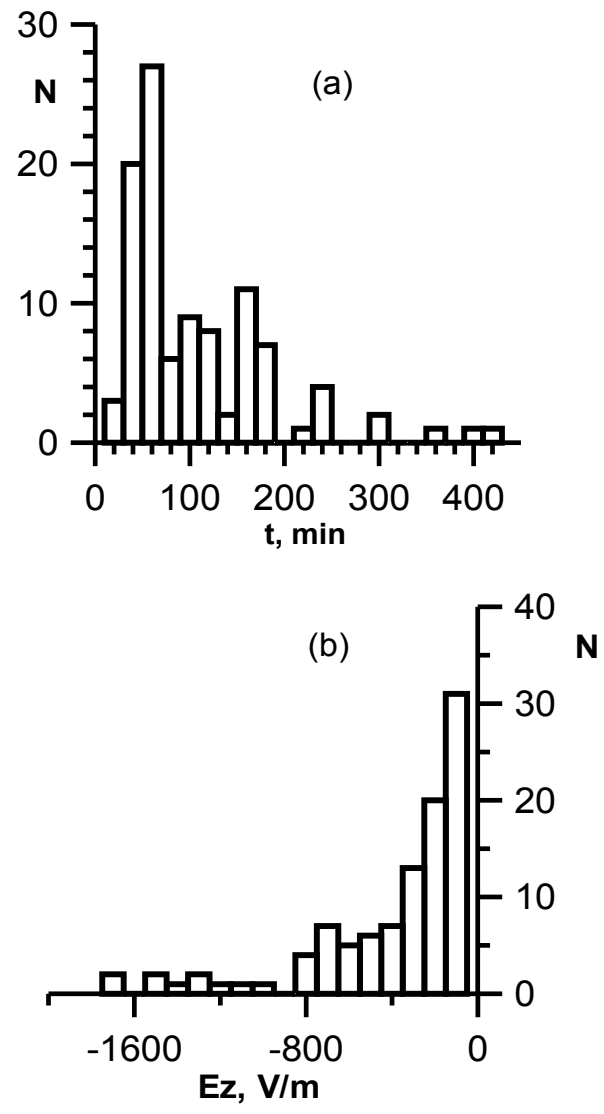

Fig. 2. Distribution of the field strength anomaly with respect to (a) bay duration and (b) decrease in strength in the bay.

anomaly duration is $40-60$ min with an additional maximum lasting $\sim 160 \mathrm{~min}$, and the most probable decrease in $E z$ is about minus (100-300) $\mathrm{V} / \mathrm{m}$. In addition, the distribution of $E z$ anomalies with respect to momentum (Fig. 3a) and energy (Fig. $3 \mathrm{~b}$ ) were constructed. The integrals $\mathrm{P}=\int E z(t) d t$ and $\mathrm{E}=\int E z^{2}(t) d t$ over the anomaly duration were taken as a bay momentum and energy, respectively. Figure 3 shows that the anomalies are concentrated in the region with small values of these parameters. The magnitudes of $E z$ anomalies in the bay as a function (a) of earthquake class and (b) of the distance between the observation point and epicenter are shown in Fig. 4. The computed correlation coefficients are 0.17 and 0.09 , respectively, which indicates that the relation between these parameters is almost absent.

\section{Discussion}

The statistical properties of the negative anomalies in the quasistatic electric field in the atmosphere within $1-24 \mathrm{~h}$ before earthquakes were considered. According to the classification by Sobolev (1993), these anomalies can be considered as short-term predictions. As was noted in (Morgounov, 2004; Sobolev, 1993), the anomalies are charac-
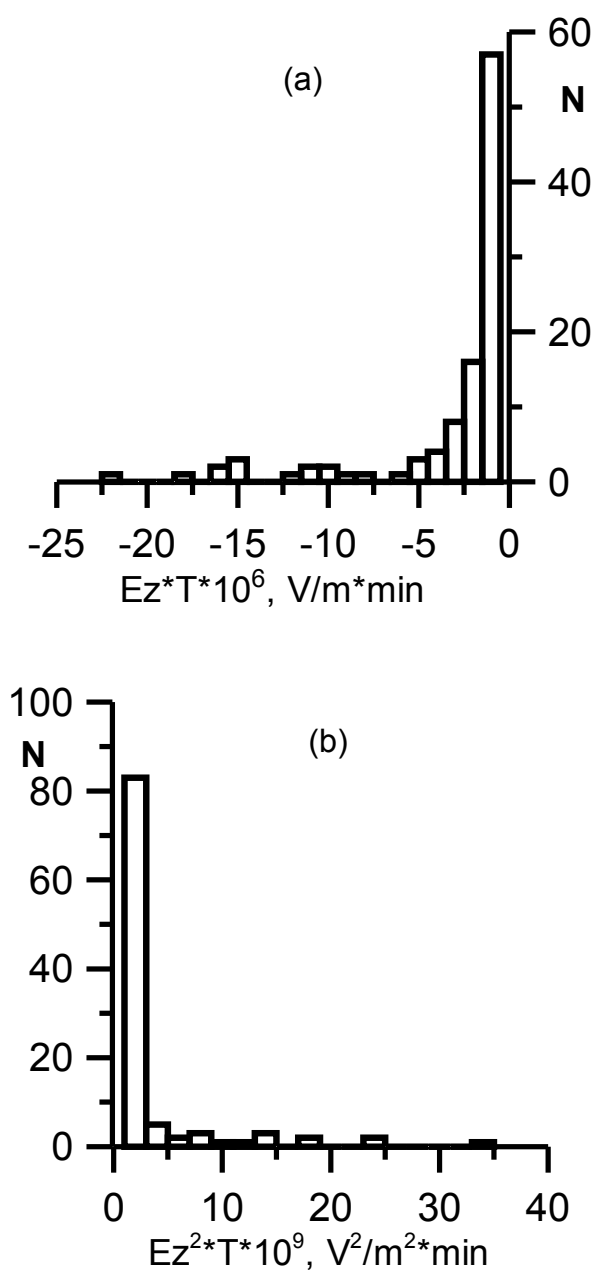

Fig. 3. Distribution of $E z$ anomalies with respect to (a) momentum and (b) energy.

terized by the absence of significant relation between such parameters as delay time of event, magnitude of anomaly, and earthquake magnitude (M). The spatial dimensions of the earthquake preparation zone does not obey the empirical formulas $r \sim e^{M}$ and $r \sim 10^{0.43 M}$ used in long-term predictions (Sidorin, 1979; Sobolev, 1993). This is explained by nonuniformity of stressed and strain processes in the Earth's crust immediately before earthquakes, which indirectly manifests itself in a mosaic structure of different geophysical parameters (e.g. see Morgounov, 2004). The absence of significant relation of an $E z$ anomaly to an earthquake magnitude and epicentral distance (see Fig. 4) can apparently be related to the spatially inhomogeneous emanation of radon, which is the main agent of the quasistatic electric field variation in the near-Earth atmosphere, into the atmosphere (Outkin et al., 1997; Outkin and Yurkov, 1998). A similar effect, i.e. the absence of a relation between the distance to the epicenter and the magnitude of two strong earthquakes on Kamchatka was observed earlier in the variations in short-period disturbances of the electrotelluric field (Sobolev and Morozov, 1970). 


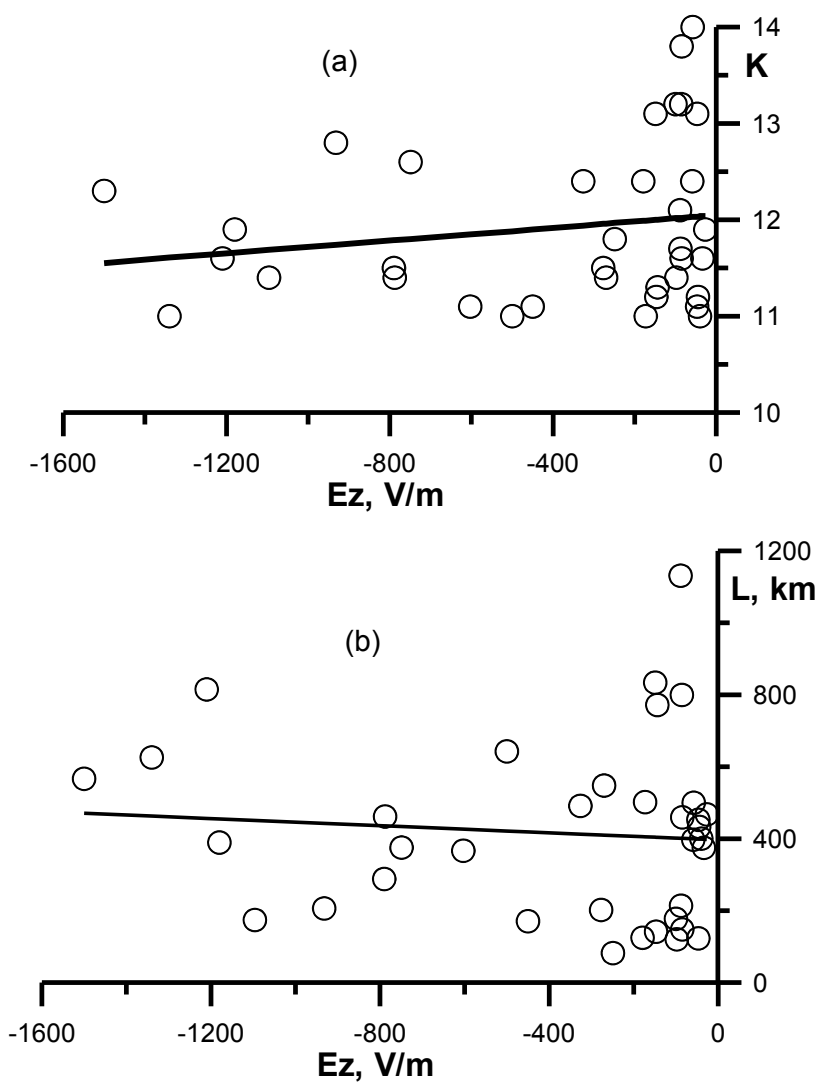

Fig. 4. The magnitude of $E z$ anomalies in a bay as a function of (a) the earthquake class and (b) the distance from the earthquake epicenter to the observation point.

The nature of the anomalies of the quasistatic electric field before earthquakes observed on Kamchatka is still unclear. A few models of this effect were proposed in the literature. The first theoretical estimates of $E z$ variations in the near-Earth atmosphere due to a change in the radon concentration were made in Pierce (1976). In all mechanisms proposed later, radon was also considered as the main agent changing conductivity of the near-Earth atmosphere and, consequently, the electric field (Rulenko, 2000, 2003; Shuleikin, 2003). The radon content of the Earth's crust and its release into atmosphere are closely related to the state of deformation processes in surface layers of the Earth in the period of active fracturing during earthquake preparation. The exception is the model (Morgunov and Maltsev, 2003), which considers the formation of polarization charges on the walls of rock fractures, generating a quasistatic field in the atmosphere in addition to the background field. A mathematical modeling of earthquake precursors in electric fields $(E x, E y, E z)$ was performed in Alekseev and Aksenov (2003). It was shown that the field magnitude $|E z|$ depends on a difference between the electrical conductivity of the air and the Earth. The latter is, in turn, governed by the conductivity of fluids filling rock pores and fractures. It is clear that the above examples of different mechanisms of anomaly formation reflect the complexity of fast tectonic processes in the Earth's crust immediately before an earthquake rather than finally reveal the nature of these anomalies.

\section{Conclusions}

A statistical analysis of 103 cases of a bay-like decrease in the magnitude of the $E z$ component of the quasistatic electric field in the near-Earth atmosphere on Kamchatka indicated the following.

1. The most probable bay duration is $40-60 \mathrm{~min}$ with an additional maximum lasting $160 \mathrm{~min}$.

2. The most probable decreases in the electric field strength are minus $(100-300 \mathrm{~V} / \mathrm{m}$. The anomalies with a magnitude of minus $800-1000 \mathrm{~V} / \mathrm{m}$ were observed extremely rarely.

3. The dependence of a bay duration and a decrease in the field strength on an earthquake class or a distance to an epicenter was not found.

4. An earthquake can occur 1-24 h after an electric field anomaly with a probability of about $36 \%$.

Edited by: M. Contadakis

Reviewed by: G. Vodinchar and another anonymous referee

\section{References}

Alekseev, A. S. and Aksenov, V. V.: On an Electric Field in an Earthquake Epicenter, Dokl. Akad. Nauk, 392(1), 106-110, 2003.

Buzevich, A. V., Cherneva, N. V., Babakhanov, I. Yu., and Smirnov, S.: Relation of the Variations in the Geomagnetic and Atmospheric Electric Fields to Seismicity against a Background of Heliomagnetospheric Processes, in: Proceedings of the 5th Russian Conference on Atmospheric Electricity, Vladimir, Russia, 3-8 September 2003, 2, 72-75, 2003.

Buzevich, A. V., Smirnov, S., Filimonov, V. I., and Firstov, P. P.: Paratunka Specialized Hardware-Software Complex for Geophysical Data Acquisition and Processing, in: SolarTerrestrial Coupling and Electromagnetic Precursors of Earthquakes, Petropavlovsk-Kamchatskii, Russia, 18-21 August 1998, 36-38, 1998a.

Buzevich, A. V. and Smirnov, S. E.: Relation of Solar Activity and Slow Variations in the Magnetic and Electric Fields to Strong Kamchatka Earthquakes, in: Solar-Terrestrial Coupling and Electromagnetic Precursors of Earthquakes, PetropavlovskKamchatskii, Russia, 18-21 August 1998, 13-14, 1998b.

Buzevich, A. V., Druzhin, G. I., Firstov, P. P., Vershinin, E. F., Smirnov, S., and Filimonov, V. I.: Heliogeophysical Effects before the Kronotsk Earthquake of December 5, 1997 M=7.7, in: Kronotsk Earthquake of December 5, 1997, on Kamchatka: 
Precursors, Features, Aftereffects, Petropavlovsk-Kamchatskii, Russia, 177-188, 1998c.

Chernyavskii, E. A.: On a Method for Deriving Diurnal Variations in the Atmospheric Electric Potential, in: Information Volume on Terrestrial Magnetism and Electricity, Moscow, Russia, 1937, 82-86, 1937.

Hao Jian-Guo, Tang Tian-Ming, and Li De-Rui: A kind of Information on Short-Term and Imminent Earthquake Precursors: Research on Atmospheric Electric Field Anomalies before Earthquakes, Acta Seismol. Sin., 11(1), 121-131, 1998.

Kondo, G.: The Variation of the Atmospheric Electric Field at the Time of Earthquake, Kakioka Magn. Obs. Mem., 13(1), 11-23, 1968.

Mikhailov, Yu. M., Mikhailova, G. A., Kapustina, O. V., Depueva, A. X., Buzevich, A. V., Druzhin, G. I., Smirnov, S., and Firstov, P. P.: Variations in Different Atmospheric and Ionospheric Parameters in the Earthquake Preparation Periods on Kamchatka: Preliminary Results, Geomagn. Aeron., 42, 769-776, 2002.

Mikhailov, Yu. M., Mikhailova, G. A., Kapustina, O. V., Buzevich, A. V., and Smirnov, S.: Variations in the Power Spectra of the Electric Field in the Near-Earth Atmosphere on Kamchatka, Geomagn. Aeron., 43, 395-401, 2003.

Morgounov, V. A.: Slip Weakening, Strain and Short-Term Preseismic Disturbances, Ann. Geophys., 47(1), 133-149, 2004.

Morgunov, V. A. and Maltsev, S. A.: Model of the Quasistationary Lithospheric Electric Field, in: Proceedings of the 5th Russian Conference on Atmospheric Electricity, Vladimir, Russia, 3-8 September 2003, vol. 2, 59-61, 2003.

Outkin, V. I., Yurkov, A. K., Krivasheev, S. V., and King Chi-Yu: Radon Behavior in Connection with Rock Bumps (Shocks) in Deep Mines and Tectonic Earthquakes, Yekaterinburg, Russia, 1997.

Pierce, E. T.: Atmospheric Electricity and Earthquake Prediction, Geophys. Res. Lett., 3(3), 185-188, 1976.

Rulenko, O. P.: Operative Earthquake Precursors in Electricity of the Near-Earth Atmosphere, Vulkanol. Seismol., 4, 57-68, 2000.

Rulenko, O. P.: Strain Sensitivity of Preseismic Negative Anomalies of the Electric Field in the Near-Earth Air, Vulkanol. Seismol., 4, 82-85, 2003.
Rulenko, O. P., Ivanov, A. V., and Shumeiko, A. V.: Short-Term Atmospheric Electric Precursor of the Kamchatka Earthquake of March 6, 1992, Dokl. Akad. Nauk, 326(6), 980-982, 1992.

Rulenko, O. P., Druzhin, G. I., and Vershinin, E. F.: Measurements of the Atmospheric Electric Field and Natural Electromagnetic Radiation before the Kamchatka Earthquake of November 13, 1993, Dokl. Akad. Nauk, 348(6), 814-816, 1996.

Shuleikin, V. N.: Variations in the Elements of the Near-Earth Atmospheric Electricity before Seismic Events: Manifestation Causes, Forms, and Scales, in: Geophysics of the 21th Century: 2002, Moscow, Russia, 396-404, 2003.

Sidorin, A. Ya.: Dependence of the Time of Earthquake Precursors on the Epicentral Distance, Dokl. Akad. Nauk SSSR, 245(4), 825-828, 1979.

Smirnov, S.: Anomalous Behavior of the Electric Field Strength before Earthquakes Observed at Paratunka Magnetic Observatory in 1997-2002, in: Proceedings of the 5th Russian Conference on Atmospheric Electricity, Vladimir, Russia, 3-8 September 2003, vol. 2, 72-75, 2003.

Smirnov, S.: Anomalous Behavior of the Electric Field Strength before Earthquakes Observed at Paratunka Magnetic Observatory in 1997-2000, in: Solar-Terrestrial Coupling and Electromagnetic Precursors of Earthquakes, Petropavlovsk-Kamchatskii, Russia, 14-19 August 2001, 48-49, 2001.

Smirnov, S.: Paratunka Specialized Geophysical HardwareSoftware Complex, in: Metrological Backgrounds of Magnetic Observations of Siberia and Far East, PetropavlovskKamchatskii, Russia, 11-16 August 2003, 7-10, 2003.

Sobolev, G. A. and Morozov, V. N.: Local Electric Field Disturbances on Kamchatka and Their Relation to Eartquakes, in: Physical Backgrounds of a Search for Earthquake Prediction Methods, Moscow, Russia, 110-121, 1970.

Sobolev, G. A.: Earthquake Prediction Backgrounds, Moscow, Russia, 1993.

Vershinin, E. F., Buzevich, A. V., Yumoto, K., et al.: Atmospheric and Ionospheric Electromagnetic Phenomena Associated with Earthquakes, Tokyo, Japan, 513-517, 1999. 